\title{
Left Ventricular Ejection Fraction Screening and Clinical Decision-making in Metastatic HER2-positive Breast Cancer
}

\author{
CRAIG MESCHER ${ }^{1,2}$, CHRISTINE DING $^{2}$, TODD DEFOR ${ }^{3}$, SUMA KONETY ${ }^{2,4}$ and ANNE BLAES ${ }^{1,2}$ \\ ${ }^{1}$ Division of Hematology Oncology, and Transplant, ${ }^{2}$ Department of Medicine, and \\ ${ }^{4}$ Division of Cardiology, University of Minnesota, Minneapolis, MN, U.S.A.; \\ ${ }^{3}$ Biostatistics Core, Masonic Cancer Center, Minneapolis, MN, U.S.A.
}

\begin{abstract}
Background: Antibodies against human epidermal growth factor receptor 2 (HER2) affect metastatic breast cancer cells and cardiac myocytes. Guidelines recommend evaluation of cardiac ejection fraction $(E F)$ every 3 months despite little supporting evidence for this need. We assessed the impact of EF screening on clinical practice. Patients and Methods: We carried out retrospective analysis of patients with HER2-positive metastatic breast cancer receiving HER2-directed therapy to assess the impact of aggressive cardiac screening on management decisions. Results: Data for 128 patients were analyzed. The median number of EF screenings in the first year of therapy was 3 (range $=1-8$ ). A total of 29 patients had an asymptomatic decrease in EF. These patients had more EF screenings, more exposure to anthracyclines or left-sided radiation, were more likely to receive a cardiology consult and have an angiotensin converting enzyme inhibitor added to their therapy. Ninety patients underwent aggressive screening; this was not associated with cessation of HER2 therapy ( $p=0.92)$. Conclusion: Routine EF screening did not have an impact on decisions regarding HER 2 therapy in patients with metastatic breast cancer. Given the known benefit of HER2 treatment, reducing the frequency of cardiac screening may be reasonable.
\end{abstract}

Breast cancer is the most common malignancy and the second most common cause of cancer mortality among woman in the United States (1). Human epidermal growth factor receptor 2 (HER2) is a transmembrane tyrosine kinase

Correspondence to: Craig Mescher, MD, University of Minnesota, Division of Hematology, Oncology, and Transplant, 420 Delaware Ave SE, MMC 480, Minneapolis, MN 55455, U.S.A. Tel: +1 6126240123, Fax: +1 6126256919, e-mail: Mesc0014@umn.edu

Key Words: HER2, metastatic breast cancer, echocardiography, ejection fraction. receptor that is overexpressed in one quarter of all breast cancer cases. Expression of HER2 by breast cancer cells is associated with a worse prognosis and more aggressive disease in the local and metastatic setting (2). The introduction of monoclonal antibodies directed against HER2 (trastuzumab, pertuzumab, and ado-trastzumab) changed the landscape for treatment of HER2-positive breast cancer, with significant improvements in survival both in the metastatic and the neoadjuvant/adjuvant setting. In heavily pretreated patients with metastatic breast cancer, progression-free and overall survival improved by 3 and 5 months, respectively (3). The use of trastuzumab for metastatic HER2-positive breast cancer extended 3-year overall survival to $50.2 \%$ (4). Meta-analysis showed that the addition of trastuzumab to improve overall survival [hazard ratio $(\mathrm{HR})=0.81$ ], with the main benefit derived when trastuzumab is used in first-line therapy, although its use in the heavily pretreated setting also extends life expectancy $(3,5)$. The addition of pertuzumab to trastuzumab in metastatic breast cancer, and the use of trastuzumab emtansine, was found to further improve median progression-free survival by an additional 6 months $(6,7)$.

HER2 is also present on cardiac myocytes. HER2 is required for myocyte development, involved in cell signaling though cellular kinases such as phosphoinositide 3-kinase and mitogen-activated protein-kinase, and thought to be important in cardiac stress response (8). Although closer to understanding how trastuzumab impacts cardiac function, the exact mechanism remains unknown (8). In the initial phase 3 study of trastuzumab, cardiotoxicity was identified as a notable side-effect occurring in up to $22 \%$ of patients receiving chemotherapy plus trastuzumab, although the majority of these occurred in patients receiving anthracycline plus trastuzumab (3). Cochrane review indicated that when trastuzumab is added to chemotherapy in the metastatic setting there is an increased relative risk (RR) of cardiotoxicity ( $R R=3.49)(5)$, although this was largely influenced by the Slamon et al. study (2). Wide ranges in the incidence of asymptomatic cardiotoxicity amongst those 
treated with trastuzumab exist, ranging from $<1 \%$ to $80 \%$ of patients (9). Dual HER2 blockade did not appear to increase the risk of reduced left ventricular ejection fraction (EF) (6).

The Food and Drug Administration (FDA) recommends screening for cardiac dysfunction every 3 months for the duration of trastuzumab therapy as well as every 6 months after cessation for 2 years. This does not include any differentiation between adjuvant therapy or therapy for metastatic disease (10). Given the significant advances in survival with the continuation of trastuzumab-based therapy, organizational guidelines have recently indicated the optimal screening interval is less clear and screening is possibly not necessary at all in the metastatic setting (11-13).

With the known benefit to survival of HER2-blocking agents such as trastuzumab in HER2-positive metastatic breast cancer and the lack of evidence-based guidelines for cardiac screening, the best approach for monitoring cardiac function is unclear. In this study, we aimed to identify the effect of aggressive cardiac screening on management decisions in women with HER2-positive metastatic breast cancer.

\section{Patients and Methods}

A retrospective study assessing the impact of aggressive cardiac screening on management of patients with HER2-positive metastatic breast cancer was performed. After approval by the Institutional Review Board (1505M71981), patients were identified through the University of Minnesota and Fairview health services i2b2 clinical data repository. All adult patients (18 years or older) treated with a HER2-blocking agent (trastuzumab, pertuzumab, or trastuzumab emtansane) were identified. Each chart was individually reviewed and all patients with metastatic disease were included.

Available records for these charts were reviewed for baseline characteristics including age, date of diagnosis of breast cancer, date of diagnosis of metastatic disease, histological type of breast cancer, and hormone receptor status. Cardiovascular risk factors were identified including hypertension, coronary artery disease, congestive heart failure, kidney disease, diabetes mellitus, smoking history, prior treatment (i.e. anthracycline use), and prior radiation therapy. Use of cardiac medications, angiotensin-converting enzyme (ACE) inhibitors, angiotensin receptor blockers, beta blockers, diuretics, and anti-arrhythmic agents at the time of diagnosis, after the cessation of HER2 directed therapy (if applicable), and at the time of last documented cardiac EF screening were collected.

Cardiac screening was identified by type of screening (transthoracic echocardiogram, radionuclide ventriculography, or magnetic resonance imaging) as well as frequency of screening and EF as reported. The number of evaluations of EF in one year was used to determine the aggressiveness of cardiac screening. Three or more evaluations of EF were considered aggressive screening, while fewer than three evaluations within one year was considered not aggressive. Patients were excluded from analysis if they did not have any evaluation of EF or report of evaluation. The primary outcome was management decisions in the setting of aggressive cardiac screening. This included cardiology consultation, medication changes, cessation or continuation of HER2-blocking therapy, and reason for changing therapy.
Categorical factors were compared between groups using the chisquare test or Fisher's exact test when expected cell counts were small. The general Wilcoxon test was used to compare continuous factors between groups. All reported $p$-values are two-sided. SAS 9.3 (SAS Institute, Cary, NC, USA) was used for all statistical analyses.

\section{Results}

Of the 147 identified patients with HER2-neu-positive metastatic breast cancer treated any time from 2011-2015, $128(87 \%)$ underwent at least one cardiac imaging study for EF screening with an available report (Table I) and were included in analysis. The average number \pm standard deviation of screenings through one year of therapy was $3.4 \pm 1.6$ per year. When averaged over 2 years, the average was 3.0 per year $(6.0 \pm 3.1$ over 2 years). Ninety patients $(61 \%)$ underwent aggressive screening. Compared to those who were not aggressively screened, those who were aggressively screened were more likely to be on beta-blockers (22\% vs. $3 \%, p=0.05)$ and diuretics $(10 \% v s .0 \%, p=0.04)$ prior to the initiation of therapy. Those who were not aggressively screened were more likely to have received adjuvant chemotherapy $(86 \%$ vs. $46 \%, p=0.02)$. There was no statistically significant difference in the remainder of baseline characteristics (Table I).

During the follow-up period, 29 patients (23\%) were found to have a decrease in left ventricular EF or the value fell below the lower limit of $50 \%$. No patient was symptomatic. These patients underwent more frequent EF screenings than patients without a decrease in EF (4.6 vs. 3.0. $p<0.01)$ and were more likely to receive a cardiology consultation ( $48 \%$ vs. $15 \%, p<0.01$ ). Eight out of 128 patients $(6 \%)$ stopped therapy due to a decrease in $\mathrm{EF}$, while 15 out of 128 (12\%) did not stop therapy. Four out of those eight patients were re-challenged with trastuzumab without further problems. Those who did not stop therapy were continued on trastuzumab without further incident. Patients who had a decreased EF were more likely to have an ACE inhibitor added to their medications (28\% vs. $10 \%$, $p=0.02)$.

Aggressive screening was more likely to identify a decrease in EF. Twenty-six of out 90 patients $(29 \%)$ undergoing aggressive screening and three out of $38(8 \%)$ who did not undergo aggressive screening went on to develop a decrease in EF identified on cardiac imaging $(p=0.01)$. While aggressive screening identified more individuals with a decline in EF, there was no difference in management with aggressive screening (Table II). Cessation of HER2-directed therapy $(41 \% v s .42 \%, p=0.92)$ did not differ between the two groups. After excluding patients who stopped trastuzumab upon enrollment into hospice, aggressive screening still did not result in a difference in cessation of therapy $(p=0.98)$. Aggressive screening was not associated 
Table I. Baseline characteristics among screened patients.

\begin{tabular}{|c|c|c|c|c|}
\hline & Total N & $\geq 3 \mathrm{EPY}$ & $<3 \mathrm{EPY}$ & $p$-Value \\
\hline Total & 128 & 90 & 38 & \\
\hline Median age at diagnosis (range) & $56(26-89)$ & $57(26-89)$ & $55(28-84)$ & 0.84 \\
\hline \multicolumn{5}{|l|}{ Comorbidities } \\
\hline Hypertension & $46(36 \%)$ & $35(39 \%)$ & $11(29 \%)$ & 0.28 \\
\hline Coronary artery disease & $5(4 \%)$ & $3(3 \%)$ & $2(5 \%)$ & 0.63 \\
\hline Congestive heart failure & $5(4 \%)$ & $3(3 \%)$ & $2(5 \%)$ & 0.63 \\
\hline \multicolumn{5}{|l|}{ Smoking history } \\
\hline Current smoker & $7(6 \%)$ & $2(5 \%)$ & $5(6 \%)$ & 0.71 \\
\hline Past smoker & $38(32 \%)$ & $24(29 \%)$ & $14(37 \%)$ & \\
\hline Nonsmoker (never/unknown) & $75(63 \%)$ & $53(65 \%)$ & $22(58 \%)$ & \\
\hline Adjuvant chemotherapy & $67(52 \%)$ & $41(46 \%)$ & $26(86 \%)$ & 0.02 \\
\hline Prior anthracycline exposure & $45(35 \%)$ & $30(33 \%)$ & $15(40 \%)$ & 0.80 \\
\hline Prior radiation exposure & $51(40 \%)$ & $32(36 \%)$ & $19(50 \%)$ & 0.13 \\
\hline Hormone receptor positive & $91(72 \%)$ & $62(70 \%)$ & $29(76 \%)$ & 0.52 \\
\hline Grade $2 / 3$ tumor & $86(97 \%)$ & $65(98 \%)$ & $21(91 \%)$ & 0.17 \\
\hline \multicolumn{5}{|l|}{ Medication at diagnosis } \\
\hline ACE-I/ARB & $27(21 \%)$ & $21(23 \%)$ & $6(16 \%)$ & 0.34 \\
\hline Anti-arrhythmic & 0 & 0 & 0 & \\
\hline Beta blocker & $23(18 \%)$ & $20(22 \%)$ & $3(8 \%)$ & 0.05 \\
\hline Diuretics & $9(7 \%)$ & $9(10 \%)$ & 0 & 0.04 \\
\hline Nitrates & 0 & 0 & 0 & \\
\hline
\end{tabular}

EPY: Echocardiograms per year; ACE-I: angiotensin-converting enzyme inhibitor; ARB: angiotensin receptor blocker.

Table II. Management decisions and aggressive screening.

\begin{tabular}{lccc}
\hline & $\geq 3$ EPY $(\mathrm{N}=90), \mathrm{n}(\%)$ & $<3$ EPY $(\mathrm{N}=38), \mathrm{n}(\%)$ & $p$-Value \\
\hline Cessation of HER2-directed therapy & $37(41 \%)$ & $16(42 \%)$ & 0.92 \\
Cardiology consultation & $22(24 \%)$ & $7(18 \%)$ & 0.46 \\
Addition of diuretic & $7(8 \%)$ & $9(24 \%)$ & 0.02 \\
Addition of ACE-I & $15(17 \%)$ & $23(8 \%)$ & 0.19 \\
Addition of beta blocker & $19(21 \%)$ & $10(26 \%)$ & 0.52 \\
\hline
\end{tabular}

EPY: Echocardiograms per year; ACE-I: angiotensin-converting enzyme inhibitor; ARB: angiotensin receptor blocker.

with differences in the addition of cardioprotective medications such as beta-blockers or ACE inhibitor; it was associated with the addition of a diuretic. Patients identified as having a decreased EF were more likely to receive a cardiology consultation $(48 \%$ vs. $15 \% p<0.01)$ and be placed on an ACE inhibitor ( $28 \%$ vs. $10 \%, p=0.02)$.

The risk of developing a decreased EF while on HER2directed therapy was associated with prior anthracycline use ( $36 \%$ vs. $15 \%, p=0.02)$ as well as left-sided radiation therapy ( $40 \%$ vs. $19 \%, p=0.05$ ). There was no statistically significant association of other risk factors (such as hypertension, coronary artery disease, or smoking history) and development of a decrease in EF (Table III).
Table III. Risk factors and association with a decline in ejection fraction $(E F)$.

\begin{tabular}{lccc}
\hline & \multicolumn{2}{c}{ EF decline } & \\
\cline { 2 - 3 } Baseline risk factors & Yes, n (\%) & No, n (\%) & \\
\hline Hypertension & $11 / 46(24)$ & $18 / 82(22)$ & 0.80 \\
Current smoker & $2 / 7(29)$ & $23 / 113(20)$ & 0.63 \\
CAD & $0 / 5(0)$ & $29 / 123(24)$ & 0.67 \\
Prior anthracycline exposure & $16 / 45(36)$ & $13 / 83(15)$ & 0.02 \\
Prior RT & $14 / 51(27)$ & $15 / 77(19)$ & 0.29 \\
Left-sided RT & $8 / 20(40)$ & $19 / 96(19)$ & 0.05 \\
\hline
\end{tabular}

CAD: Coronary artery disease; radiotherapy: radiation therapy; ACE-I: angiotensin-converting enzyme inhibitor. 


\section{Discussion}

We found that aggressive cardiac screening during treatment with HER2-directed therapy might have identified more patients with decreased EF. Aggressive cardiac screening, however, did not significantly affect the management of these patients; these patients remained on trastuzumab-based therapy without complication. Screening for cardiac dysfunction has become the standard approach while treating with HER2-blocking agents due to the identification of cardiotoxicity in the initial trials and FDA labeling $(3,10)$. Given the dramatic improvement in survival with the use of trastuzumab-based therapies in the metastatic breast cancer setting, routine cardiac monitoring every 3 months in average-risk women with metastatic HER2-positive breast cancer may not be needed.

In our patient population, $23 \%$ of patients had an asymptomatic decrease in EF, while no patient had a severe or symptomatic decrease in EF. The majority of these decreases were identified as a result of aggressive screening. The prevalence of decline in EF in our study is consistent with that reported in a meta-analysis. The range of asymptomatic or mild decrease in EF ranged from $1 \%$ to as high as $80 \%$, with the majority of analyzed studies finding $20-40 \%$ of patients with decreased EF (9). In addition, an adjuvant trial of patients treated with trastuzumab and chemotherapy identified as many as $18 \%$ of patients with decreased EF, with the highest risk being found in patients treated with anthracyclines (14). Although aggressive screening with serial cardiac imaging every 3 months identified more patients with asymptomatic decreases in EF, it did not affect management decisions such as referral to cardiology or stopping trastuzumab therapy. The addition of a diuretic to those aggressively screened is nonspecific because such patients can become fluid overloaded for reasons independent of cardiac function as a result of their treatment or malignancy.

In looking at cardiac risk factors for a decline in EF, there was a significant association with anthracycline use and prior left-sided radiation therapy. This is consistent with previously reported data in which prior anthracycline use and radiation therapy were associated with a decrease an EF ( $\mathrm{HR}=2.49$ and $\mathrm{HR}=1.83$, respectively) (15). Coronary artery disease (CAD) was not identified as a risk factor for HER2directed decreases in EF in our study, while CAD was shown to be an associated risk factor for EF decrease in HER2treated patients $(H R=2.54)(15)$. This is likely because our study had a small number of patients with known CAD.

This study reflects what is seen in general clinical practice and therefore provides insight into what oncologists are doing outside of formal clinical studies. In our population, the majority of patients underwent aggressive screening with 90 out of the 128 patients screened having at least three evaluations per year as per FDA guidelines. Given that aggressive screening with echocardiography or radionuclide ventriculography every 3 months did not change clinical management or outcomes in patients with metastatic HER2positive breast cancer treated with trastuzumab-based therapy, it may be reasonable to consider aggressive cardiac screening every 3 months in high-risk patients only, and pursue less intensive cardiac screening such as every 4 to 6 months in non-high risk patients. It has been estimated that reducing the number of screening echocardiograms by one per person could save $\$ 13$ million annually (16). The American College of Cardiology and the American Society of Clinical Oncology are currently in the process of identifying optimal screening recommendations (12).

A study suggested that in clinical practice aggressive cardiac screening in patients on trastuzumab-based therapy was actually only being performed $36 \%$ of the time (17). Our study would suggest that aggressive screening may truly only be needed in a high-risk subset of individuals with metastatic HER2-positive breast cancer as indicated by higher risk groups such as those with prior exposure to anthracyclines and previous left-sided radiation having increased risk of developing heart failure. It has been shown in the adjuvant setting that early discontinuation of trastuzumab due to cardiac dysfunction prior to the recommended 1 year of treatment led to an increased risk of non-heart failureassociated death and reduced overall survival compared to those who completed a year of adjuvant therapy (18). With the known benefit, early discontinuation of trastuzumabbased therapy in the metastatic setting could also significantly alter patient survival and continuation of therapy should therefore be considered.

One limitation of this study is its retrospective nature. In addition, there was a reliance on electronic medical record documentation of comorbidities and medication administration to identify changes in therapy. The size of our sample population was small and therefore the identification of particular associations is more difficult. Despite these limitations, our study suggests aggressive cardiac screening every 3 months in low-risk individuals with metastatic HER2-positive breast cancer may not be needed.

Clearer guidelines and evidence will be required to optimize screening particularly as the use of HER2-blocking agents broadens to other cancer types such as gastric and colorectal cancer. Balancing the risks and side-effects of cancer treatments with their known benefit is imperative in patients with metastatic cancer as the treatment can provide a meaningful prolongation of life. While some cardiologist argue oncologists are not identifying cardiovascular changes in a diligent manner, optimizing screening practices to those with known risk factors, such as prior anthracycline use, could be of benefit and result in more uniformly adapted practice (19). 
In conclusion, although HER2-directed therapy is associated with increase cardiotoxicity, aggressive screening for cardiac dysfunction was not found to change management decisions in clinical practice in female patients with metastatic breast cancer. In providing cost-effective medicine, it would be reasonable to consider reducing the interval of cardiac screening in the setting of metastatic HER2-positive breast cancer, particularly in non-high-risk patients, due to the known benefit of HER2-blocking treatments.

\section{References}

1 U.S. Cancer Statistics Working Group. United States Cancer Statistics: 1999-2013 Incidence and Mortality Web-based Report. Atlanta (GA): Department of Health and Human Services, Centers for Disease Control and Prevention and National Cancer Institute, 2016.

2 Slamon DJ, Clark GM, Wong SG, Levin WJ, Ullrich A and McGuire WL: Human breast cancer: correlation of relapse and survival with amplification of the HER-2/neu oncogene. Science 235: 177-182,1987.

3 Slamon DJ, Leyland-Jones B, Shak S, Fuchs H, Paton V, Bajamonde A, Fleming T, Eiermann W, Wolter J, Pegram M, Baselga $J$ and Norton L: Use of chemotherapy plus a monoclonal antibody against HER2 for metastatic breast cancer that overexpresses HER2. N Engl J Med 344: 783-792, 2001.

4 Rossi M, Carioli G, Bonifazi M, Zambelli A, Franchi M, Moja L, Zambon A, Corrao G, La Vecchia C, Zocchetti C and Negri E: Trastuzumab for HER2+ metastatic breast cancer in clinical practice: Cardiotoxicity and overall survival. Eur J Cancer 52: 41-49, 2016

5 Balduzzi S, Mantarro S, Guarneri V, Tagliabue L, Pistotti V, Moja L and D'Amico R: Trastuzumab-containing regimens for metastatic breast cancer. Cochrane Database Syst Rev 12(6): CD006242, 2014

6 Baselga J, Cortes J, Kim SB, Im SA, Hegg R, Im YH, Roman L, Pedrini JL, Pienkowski T, Knott A, Clark E, Benyunes MC, Ross G and Swain SM: Pertuzumab plus trastuzumab plus docetaxel for metastatic breast cancer. N Engl J Med 366: 109$119,2012$.

7 Verma S, Miles D, Gianni L, Krop IE, Welslau M, Baselga J, Pegram M, Oh DY, Dieras V, Guardino E, Fang L, Lu MW, Olsen, S, Blackwell K and EMILIA Study Group 2012: Trastuzumab emtansine for HER2-positive advanced breast cancer. N Engl J Med 367: 1783-1791, 2012.

8 Cote GM, Sawyer DB and Chabner BA: ERBB2 inhibition and heart failure. N Engl J Med 367: 2150-2153, 2012.

9 Mantarro S, Rossi M, Bonifazi M, D'Amico R, Blandizzi C, La Vecchia C, Negri E and Moja L: Risk of severe cardiotoxicity following treatment with trastuzumab: a meta-analysis of randomized and cohort studies of 29,000 women with breast cancer. Intern Emerg Med 11: 123-140, 2016.
10 Herceptin(R) [package insert]. Genentech, Inc., San Francisco, CA, USA, 1998.

11 Curigliano G, Cardinale D, Suter T, Plataniotis G, de Azambuja E, Sandri MT, Criscitiello C, Goldhirsch A, Cipolla C and Roila F: Cardiovascular toxicity induced by chemotherapy, targeted agents and radiotherapy: ESMO Clinical Practice Guidelines. Ann Oncol 23: 155-66, 2012.

12 Dang C, Guo H, Najita J, Yardley D, Marcom K, Albain K, Rugo H, Miller K, Ellis M, Shapira I, Wolff AC, Carey LA, Moy B, Groarke J, Moslehi J, Krop I, Burstein HJ, Hudis C, Winer EP and Tolaney SM: Cardiac outcomes of patients receiving adjuvant weekly paclitaxel and trastuzumab for node-negative, ERBB2-positive breast cancer. JAMA Onc 2: 29-36, 2016.

13 National Comprehensive Cancer Network (NCCN). NCCN Clinical Practice Guidelines in Oncology. Breast Cancer Version 2.2016. National Comprehensive Cancer Network, 2016.

14 Slamon D, Eiermann W, Robert N, Pienkowski T, Martin M, Press M, Mackey J, Glaspy J, Chan A, Pawlicki M, Pinter T, Valero V, Liu MC, Sauter G, von Minckwitz G, Visco F, Bee V, Buyse M, Bendahmane B, Tabah-Fisch I, Lindsay MA, Riva A and Crown J: Adjuvant trastuzumab in HER2-positive breast cancer. N Eng J Med 365: 1273-1283, 2011.

15 Xue J, Jiang Z, Qi F, Lv S, Zhang S, Wang T and Zhang X: Risk of trastuzumab-related cardiotoxicity in early breast cancer patients: a prospective observational study. J Breast Cancer 17: 363-369, 2014.

16 Davis CC, Zelnak A, Eley JW, Goldstein DA, Switchenko JM and McKibbin T: Clinical utility of routine cardiac monitoring in breast cancer patients receiving trastuzumab. Ann Pharmacother 50: 712-717, 2016.

17 Chavez-MacGregor M, Niu J, Zhang N, Elting LS, Smith BD, Banchs J, Hortobagyi GN and Giordano SH: Cardiac monitoring during adjuvant trastuzumab-based chemotherapy among older patients with breast cancer. J Clin Oncol 33: 2176-2183, 2015.

18 Gong IY, Verma S, Yan AT, Ko DT, Earle CC, Tomlinson GA, Trudeau ME, Krahn MD, Krzyzanowska MK, Brezden-Masley CB, Gavura S, Peacock S and Chan KK: Long-term cardiovascular outcomes and overall survival of early-stage breast cancer patients with early discontinuation of trastuzumab: a population-based study. Breast Cancer Res Treat 157: 535-544, 2016.

19 Yoon GJ, Telli ML, Kao DP, Matsuda KY, Carlson RW and Witteles RM: Left ventricular dysfunction in patients receiving cardiotoxic cancer therapies: Are clinicians responding optimally? J Am Coll Cardiol 56: 1644-1650, 2010.
Received May 5, 2017

Revised May 18, 2017

Accepted May 19, 2017 